\title{
Effect of space charge on bunch compression near the transition
}

\author{
G. Franchetti, I. Hofmann, and G. Rumolo \\ GSI, Darmstadt, Germany \\ (Received 7 April 2000; published 28 August 2000)
}

\begin{abstract}
It is shown that energy conservation in the longitudinal envelope equation can be used to derive analytic expressions to model fast bunch compression and the effect of space charge in terms of a dimensionless Coulomb parameter $\Sigma(\propto 1 / \eta$, with $\eta$ the slip factor). For small $|\eta|$ (below transition, hence $\Sigma \gg 1$ ), the rf voltage required is nearly independent of $\eta$ and dominated by space charge repulsion. The extra voltage generates the coherent momentum spread $\delta \propto 1 / \sqrt{|\eta|}$ required to compensate the increasing space charge force gradient during compression. This sets a clear limit to the useful approach to transition. An $\eta$ jump scheme is discussed to minimize this effect. Particle-in-cell computer simulation confirms the validity of our results also for more realistic beam distributions. Noticeable tails in momentum space due to the nonlinear space charge force are found for Gaussian line density bunches and $\Sigma \gg 1$.
\end{abstract}

PACS numbers: 29.27.Bd

\section{INTRODUCTION}

Bunch compression against space charge is an important issue in applications such as high-power proton drivers (for neutrons, muon or neutrino facilities, etc.) or highintensity heavy ion rings as considered for radioactive beam facilities or inertial fusion. In the absence of space charge it may seem attractive (for particles of a few $\mathrm{GeV} / \mathrm{u}$ ) to lower the stringent rf voltage requirements by working closer to transition energy. For high-intensity bunches, and below transition, this requires careful study since space charge effects become enhanced when approaching transition energy.

In this work we refer to the scheme of a $90^{\circ}$ bunch rotation by a fast jump of the rf voltage (alternatively, an $\eta$ jump at fixed voltage to increase the bucket height), whereas adiabatic compression is not considered as it may be impractical due to the high voltage requirement. For a quantitative analysis it is convenient to use the standard longitudinal envelope equation. It is a self-consistent model of a bunch with parabolic current profile and "square root" distribution in phase space [1] provided that the bunch is sufficiently long, in terms of the beam pipe diameter, to yield a linearly rising space charge force. We note that part of the work presented here (the drift approximation) follows earlier derivations for $|\eta| \approx 1$ in the context of heavy ion fusion compression [2,3]. Following the notation of Ref. [4], and using the distance $s$ as an independent variable, we have for the envelope $z_{m}$

$$
z_{m}^{\prime \prime}+k_{z 0}^{2} z_{m}-\frac{K_{L}}{z_{m}^{2}}-\frac{\epsilon_{L}^{2}}{z_{m}^{3}}=0,
$$

with $k_{z 0}^{2}=e Z V h \eta /\left(2 \pi R^{2} \gamma \beta^{2} A m c^{2}\right)$ the linearized $\mathrm{rf}$ focusing force constant for a voltage $V$ at harmonic $h$, the longitudinal perveance $K_{L}=-3 g N\left(Z^{2} / A\right) r_{p} \eta /$ $\left(2 \beta^{2} \gamma^{3}\right)$, the $g$ factor

$$
g=0.5+2 \ln \left(R_{p} / R_{b}\right)
$$

( $R_{p}$ and $R_{b}$ the pipe and beam radii), $N$ the bunch intensity, $Z$ the charge of an ion with mass $A$, and $r_{p} \approx 1.53 \times$ $10^{-18} \mathrm{~m}$. The longitudinal emittance is defined as area in $z, z^{\prime}$, hence $\epsilon_{L}=|\eta| z_{m}\left(\delta p / p_{0}\right)_{0}$, with the slip factor given by $\eta=1 / \gamma_{t}^{2}-1 / \gamma^{2}$. $\left(\delta p / p_{0}\right)_{0}$ is as shown in Fig. 1, which becomes the maximum momentum deviation $\Delta p / p_{0}$ for an upright ellipse. It is noted that this emittance is not an invariant if $\eta$ changes.

Some caution is required when using this equation near transition energy. We assume $|\eta|$ is sufficiently large that corrections to the momentum compaction factor from $\Delta p / p$ or a shift in the betatron tune by space charge can be ignored (it is shown here that space charge may eliminate the reasons that such small values of $|\eta|$ should be considered). An extension of our analysis to include the case of arbitrarily small $|\eta|$ or an $\eta$ spread is left to future studies.

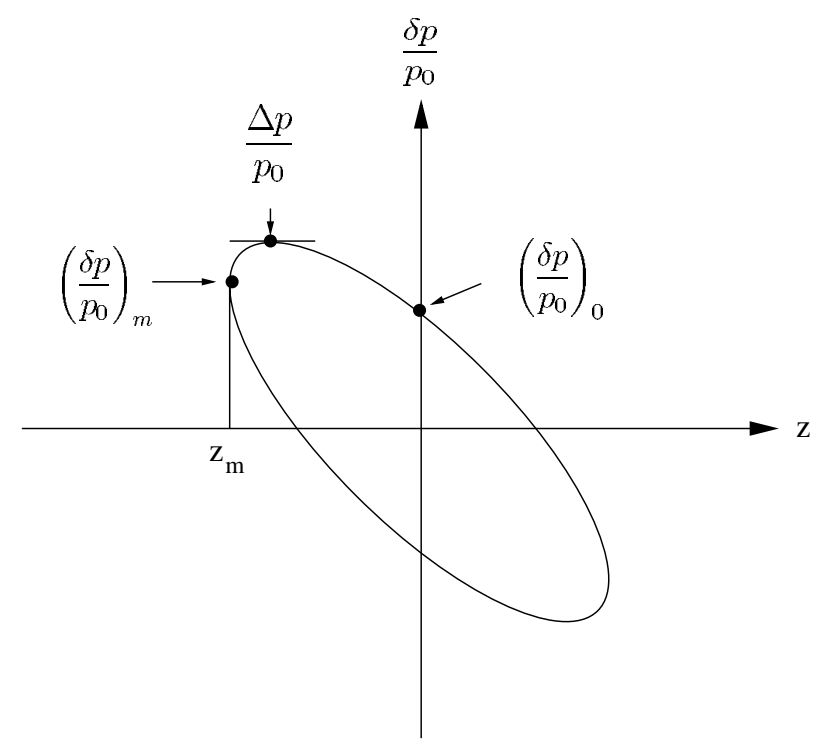

FIG. 1. Definition of parameters for a rotated ellipse in the fast compression scheme.

(C) 2000 The American Physical Society

084201-1 
The plan of the paper is the following: In Sec. II we discuss energy conservation, which is applied in Sec. III to derive analytical expressions for the coherent momentum spread, rf voltage, and compression time and a comparison with examples of numerical integration of the envelope equation. Results are compared with computer simulation using a self-consistent particle-in-cell code in Sec. IV.

\section{ENERGY PRINCIPLE AND SPACE CHARGE PARAMETER $\Sigma$}

Multiplying Eq. (1) by $z_{m}^{\prime}$ and integrating it with respect to $s$ we obtain a longitudinal invariant $I_{L}$ which expresses energy conservation

$$
\frac{z_{m}^{\prime 2}}{2}+\frac{k_{z 0}^{2}}{2} z_{m}^{2}+\frac{K_{L}}{z_{m}}+\frac{\epsilon_{L}^{2}}{2 z_{m}^{2}}=I_{L} .
$$

The first term (ignoring mass) is equivalent to a kinetic energy of coherent motion, the second term the "rf potential energy," the third term the "Coulomb energy" of the bunch, and the fourth term the "incoherent kinetic energy" expressed by the emittance. Noting the dependence on $\eta$ of the space charge and emittance terms in the energy principle, it is obvious that in the limit of $|\eta| \rightarrow 0$ the space charge term by far dominates the emittance term, and compression therefore only deals with space charge.

Here we introduce a dimensionless space charge parameter $\Sigma$, which is the ratio of the Coulomb energy over the "thermal energy" (generalizing the nomenclature suggested in Ref. [3], where $|\eta| \approx 1$ was assumed and a factor of $1 / 2$ was included in the definition of $\Sigma$ ),

$$
\Sigma \equiv \frac{2 K_{L} z_{m}}{\epsilon_{L}^{2}} .
$$

We observe that, due to the invariance of $\epsilon_{L}$ (for fixed $\eta$ ), $\Sigma \propto z_{m}$, hence space charge is even more dominant for the uncompressed bunch. Below transition $\Sigma>0$, which corresponds to a repulsive space charge force, whereas the changing sign above transition makes space charge effectively attractive (see also Sec. III G).

For the rotated ellipse in the plane $\left(z, \delta p / p_{0}\right)$, we define $\pm\left(\delta p / p_{0}\right)_{m}$ as the momentum offset of the bunch ends and $\pm\left(\delta p / p_{0}\right)_{0}$ as the maximum momentum deviation in the bunch center (see Fig. 1). The total momentum spread (abbreviated by $\Delta$ in the following) is then subject to the relationship $\Delta^{2} \equiv\left(\Delta p / p_{0}\right)^{2}=\left(\delta p / p_{0}\right)_{m}^{2}+$ $\left(\delta p / p_{0}\right)_{0}^{2}$. Using $z^{\prime}=-\eta \delta p / p_{0}$ we find

$$
\Delta=\sqrt{\left(\frac{z_{m}^{\prime}}{\eta}\right)^{2}+\left(\frac{\epsilon_{L}}{\eta z_{m}}\right)^{2}} .
$$

By inserting Eq. (5) in Eq. (3) we express the energy principle directly in terms of the total momentum spread

$$
\frac{1}{2} \eta^{2} \Delta^{2}+\frac{k_{z 0}^{2}}{2} z_{m}^{2}+\frac{K_{L}}{z_{m}}=I_{L}
$$

\section{COMPRESSION MECHANISM}

For the compression by the factor $\chi$, we start from the initial length $z_{i}$ and initial momentum spread $\Delta_{i}$ and obtain a final $z_{f}=\chi z_{i}$. Because of the invariance of the longitudinal phase area the final momentum spread results as $\Delta_{f}=\Delta_{i} / \chi$ for the assumed ideal compression. Without space charge the reduction of the rf potential energy is compensated by the increasing momentum spread.

With sufficiently strong space charge (and assuming we are below transition), the total momentum spread adopts its maximum before the end of compression. This is the case at the point of compression where the repulsive Coulomb force gradient equals the rf force gradient; in the remaining part of the rotation the dominating Coulomb force reduces this momentum spread to the final one. The intermediate extra coherent spread is needed to balance the dominating space charge repulsion while compression is completed. An alternative to avoid this extra coherent spread - not further considered here-would be an rf gradient increasing during compression to match the space charge repulsion.

The bunch length $z_{1}$ where the maximum coherent momentum spread $\Delta_{\text {max }}$ occurs is obtained by taking the derivative of Eq. (6) with respect to $z_{m}$ and requiring $\Delta^{\prime}=$ 0 , which results in $z_{1}=\left(K_{L} / k_{z 0}^{2}\right)^{1 / 3}$. Using Eq. (6) we then find

$$
\frac{1}{2} \eta^{2} \Delta_{\max }^{2}+\frac{3}{2}\left(k_{z 0} K_{L}\right)^{2 / 3}=I_{L},
$$

which is physical only if $z_{f} \leq z_{1} \leq z_{i}$. In the opposite case we are in the regime, where the rf force gradient always exceeds the Coulomb force gradient, hence the final momentum spread adopts its maximum at the end of the compression and $\Delta_{\max }=\Delta_{f}$. This regime of rf gradient dominance is characterized by a condition for the initial space charge parameter

$$
\Sigma_{i} \leq \frac{2 \chi(1+\chi)}{1+\chi-2 \chi^{2}} .
$$

This implies that the need for an extra coherent momentum spread vanishes for sufficiently weak compression. Note that the second order derivative of $\Delta$ shows that the extremum calculated at $z_{1}$ is always a maximum.

Using Eq. (7) and calculating $I_{L}$ from the parameters at the final compression, we find for the maximum momentum spread during bunch compression (except for the $\mathrm{rf}$ gradient dominated regime)

$$
\begin{aligned}
\frac{1}{2} \eta^{2} \Delta_{\max }^{2}+\frac{3}{2} & \left(k_{z 0} K_{L}\right)^{2 / 3} \\
& =\frac{1}{2} \eta^{2} \Delta_{f}^{2}+\frac{k_{z 0}^{2}}{2} z_{f}^{2}+\frac{K_{L}}{z_{f}} .
\end{aligned}
$$

\section{A. Drift rotation}

Here we assume the ellipse is already rotated to a sufficiently large initial $\Delta_{i}$; thereafter the rotation is 
completed in the absence of (or with negligible) rf force, hence $k_{z 0}=0$. The actually required initial $\Delta_{i}$ to achieve a drift compression with ratio $\chi$ follows directly from Eq. (6) by comparing quantities at the beginning (i) and end (f) of the drift. Noting that $\Sigma_{f}=\chi \Sigma_{i}$ we find

$$
\Delta_{\max }^{2}=\Delta_{f}^{2}\left[1+\Sigma_{f}(1-\chi)\right]=\Delta_{f}^{2}\left[1+\Sigma_{i} \chi(1-\chi)\right] .
$$

A similar result, ignoring $\eta$, was derived in Ref. [2]. It is noted that the second term on the right-hand side results from the extra "energy" needed to compress against space charge. The relative weight of this term in determining $\Delta_{i}$ shrinks with larger compression ratio.

\section{B. rf rotation}

To calculate $\Delta_{\max }$ for the rf rotation below transition we use Eq. (9) and write it in similar form as Eq. (10):

$$
\Delta_{\max }^{2}=\Delta_{f}^{2}\left[1+\lambda \Sigma_{f}(1-\chi)\right],
$$

where $\lambda$ is expressed in terms of $\chi$ and $\Sigma_{i}$,

$$
\lambda=\frac{1+\frac{\chi^{2}}{1+\chi}+\frac{\chi}{\Sigma_{i}}-\frac{3}{2}\left(\frac{2 \chi^{2}}{1+\chi}+2 \frac{\chi}{\Sigma_{i}}\right)^{1 / 3}}{1-\chi} .
$$

We generally have $0<\lambda<1$, with $\lambda \rightarrow 1$ for $\chi \rightarrow 0$.

In the regime of large space charge with $\Sigma_{i} \gg 1 / \chi$, $\lambda$ becomes independent from $\Sigma_{i}$ and we obtain a useful universal curve for $\lambda$ shown in Fig. 2,

$$
\lambda=\frac{1+\frac{\chi^{2}}{1+\chi}-\frac{3}{2}\left(\frac{2 \chi^{2}}{1+\chi}\right)^{1 / 3}}{1-\chi} .
$$

One finds that for $\Sigma_{i}$ of the order of 100 or larger, Eq. (13) is quite accurate for all values of $\chi$; for smaller values of $\Sigma_{i}$ Eq. (13) overestimates the exact $\lambda$.

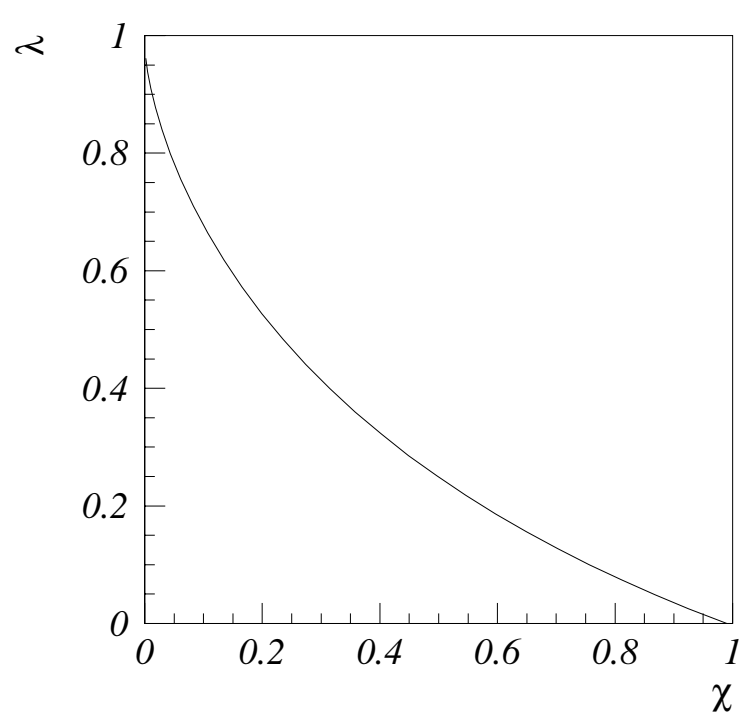

FIG. 2. The $\lambda$ factor according to Eq. (13).
It is noted that, in the limit of strong compression, Eq. (11) approaches the drift compression formula as one might expect. For not too strong compression $\left(\chi^{-1}<\right.$ $3 \cdots 4$ ), one finds that Eq. (13) is well approximated by the quadratic formula

$$
\lambda_{2}=3 / 8(1-\chi)+1 / 4(1-\chi)^{2} .
$$

\section{C. rf voltage requirements}

By using Eq. (3) at the beginning and at the end of the rf rotation we obtain the required $\mathrm{rf}$ focusing constant as

$$
k_{z 0}^{2}=\frac{|\eta|^{2} \Delta_{i}^{2}}{z_{i}^{2} \chi^{2}}\left[1+\frac{\Sigma_{f}}{(1+\chi)}\right] \text {. }
$$

The force constant to hold the initial bunch stationary (matched rf bucket) is readily obtained by inserting $\chi=1$, hence

$$
k_{z 0}^{2}=\frac{|\eta|^{2} \Delta_{i}^{2}}{z_{i}^{2}}\left[1+\Sigma_{i} / 2\right]
$$

which indicates that the holding potential is even more controlled by space charge than the one for compression. These equations can be used also above transition, where the rf gradients are reduced by space charge.

\section{Compression time}

The additional $\mathrm{rf}$ voltage reduces the time (or distance) required for the bunch rotation. In the absence of space charge the distance for the $90^{\circ}$ rotation results readily from the single particle tune as $\Delta s=\pi z_{i}^{2} \chi /\left(2 \epsilon_{L}\right)$. With space charge the fast compression is not a $90^{\circ}$ rotation for the single particles, but rather a half-period of the envelope from a maximum to a minimum. For a small envelope modulation $(\chi \approx 1)$, it is straightforward to use Eq. (1) with Eq. (16) and calculate the distance required for such a half-period as $\Delta s=\pi z_{i}^{2} /\left(2 \epsilon_{L} \sqrt{1+3 \Sigma_{i} / 8}\right)$. Since the distance required for a $90^{\circ}$ rotation of the single particle in the stationary bunch with space charge is $\pi z_{i}^{2} /\left(2 \epsilon_{L}\right)$, we notice that the single particle motion is slower than the coherent motion by the above square root factor. For significant compression including space charge we cannot find an exact analytical expression. The limiting expressions for $\Sigma=0$ and $\chi=1$ suggest, however, that an approximate expression could be of the form

$$
\Delta s \approx \pi z_{i}^{2} \chi /\left(2 \epsilon_{L} \sqrt{1+3 / 8 \Sigma_{i} \chi^{2 / 3}}\right),
$$

which has the feature of approaching the small envelope modulation result for $\chi \approx 1$, as well as the zero space charge result for infinite compression when the required voltage is again independent of space charge according to Eq. (15) $\left(\Sigma_{f} \propto \chi \approx 0\right)$. Among various powers of $\chi$ we have found that $2 / 3$ fits best the exact results from numerical integration. The error for $\chi>0.1$ is found 
below 5\%; in the examples presented in Sec. III H it is actually much smaller.

\section{E. Scaling with $\eta$}

By noting $V \propto k_{z 0}^{2} /|\eta|$ and that $\Sigma \eta$ is independent of $\eta$, we readily find, for the dependence of the compression rf voltage on $\eta$ and $\chi$,

$$
V \propto \frac{1}{\chi^{2}}\left[\eta+\frac{\Sigma_{f} \eta}{(1+\chi)}\right] .
$$

The obvious advantage of small $|\eta|$ disappears once the space charge regime is entered, where the second $(\eta$ independent) term dominates. The crossover where both terms are equally large (doubling of voltage) occurs for a value of $\eta$ such that

$$
\Sigma_{f}=1+\chi
$$

holds. The intermediate coherent momentum spread at this threshold is given by $\Delta_{\max } / \Delta_{f}=\sqrt{2-\chi^{2}}$. Further approaching transition at the most lowers the voltage by a factor of 2, but leads to an increasing coherent spread according to Eq. (11), which might result in an acceptance conflict. This is not surprising since the Coulomb repulsion is a real force, which needs to be counteracted by an equally large rf force gradient.

\section{F. Two-step compression}

Our findings suggest that due to space charge repulsion the rf voltage cannot be reduced by pushing towards small $|\eta|$, as is frequently suggested. This insensitivity of the space charge compression voltage to $|\eta| \rightarrow 0$ can be avoided, in principle, by first carrying out at small $|\eta|$ a rotation to $\Delta_{1} \approx \Delta_{f}$ with only a weak compression ratio $\chi_{1}$. The second step is a jump to sufficiently larger $|\eta|$ for the remaining (major) part of the compression such that $\Sigma$ is significantly reduced. The actual $\Delta_{1}$ required can be easily estimated by applying the drift approximation according to Eq. (10), which is justified since the rf voltage is of reduced effect at the large $|\eta|$.

For a simple estimate we now define $\lambda_{1}$ as the linearized term of Eq. (14) and insert it into Eq. (11). The resulting quadratic equation can be solved easily to calculate the required precompression

$$
\chi_{1}=1+\frac{\alpha}{2}-\sqrt{\left(1+\frac{\alpha}{2}\right)^{2}-1},
$$

with $\alpha \equiv 8 / 3\left(\Delta_{1} / \Delta_{i}\right)^{2} / \Sigma_{i}$. Using $\chi_{1}$ we can readily calculate $k_{z 0}$ from Eq. (15) and thus the precompression voltage $V_{\text {prec }}$. It is convenient to express it in units of the stationary holding voltage for the initial bunch from Eq. (16),

$$
\left(V_{\text {prec }} / V_{\text {stat }}\right)=\frac{2}{\chi_{1}\left(1+\chi_{1}\right)},
$$

which approaches unity for $|\eta| \rightarrow 0$. Hence in this limit the required $\mathrm{rf}$ voltage is $\chi(1+\chi) / 2$ times the voltage needed for compression at constant $\eta$.

\section{G. Self-focusing above transition}

Above transition $\Sigma<0$, hence space charge is attractive and enhances the applied potential according to Eq. (15). This equation [equivalently, Eq. (10) with $\Delta_{i}=0$ ] suggests "self-focusing" by the factor $\chi$, without any applied rf voltage $\left(k_{z 0}=0\right)$, if $\eta$ is chosen to satisfy

$$
\Sigma_{i, \mathrm{sf}}=-\frac{(1+\chi)}{\chi} .
$$

However, the self-focusing effect is not limited to the envelope motion, but also applies to all higher order modes (note that the envelope motion is a quadrupole mode in the longitudinal phase plane) usually called "negative mass instability." Since the growth rate increases with the mode number, the instability on the shortest wavelengths is expected to suppress self-focusing of the bunch length.

We note here that our space charge parameter $\Sigma$ is equivalent (besides a numerical factor $\approx 1$ ) to the dimensionless "impedance" parameter $U$ (see Ref. [5]) used to describe the threshold of onset of the coasting beam longitudinal instability ("local Keil-Schnell" or "Boussard" criterion) if the impedance is assumed to be the "space charge impedance" $Z_{n} / n=377 g /\left(2 \beta \gamma^{2}\right)$. The criterion $|U| \geqslant 1$, here applied locally to the bunch center, marks transition to the negative mass instability. Bunches with initial $\Sigma \gtrsim 1$ are thus subject to this instability unless the rotation is made fast enough to suppress its effect.

\section{H. Numerical examples}

As an example, we take a bunch of $10^{13}$ protons at $1.5 \mathrm{GeV}, \Delta_{i}=0.002, z_{i}=5 \mathrm{~m}$, and $\Delta_{f}=0.01, z_{f}=$ $1 \mathrm{~m}$ and assume $R_{p} / R_{b}=5$ (hence $g=3.7$ ). We find that the compression voltage $V$ is doubled by space charge for $\Sigma_{i}=6$, hence $\eta=-0.095$, and the coherent momentum spread is enhanced over the final momentum spread by a factor of 1.22. According to Eq. (16), holding of the stationary bunch prior to compression requires a voltage four times larger than ignoring space charge for the above parameters. For $\eta=-0.001$ the compression voltage is practically half as large, but the coherent momentum spread adopts a value as high as 0.07 , which is not practical.

We further assume a ring of $150 \mathrm{~m}$ radius and rf harmonic $h=24$, which allows us to determine voltages (per turn) and revolution times (in turns). Below we list a number of cases using variable $\eta$ with exact values for $\Delta_{\max } / \Delta_{f}$ and, in parentheses, the approximations using Eq. (13). 

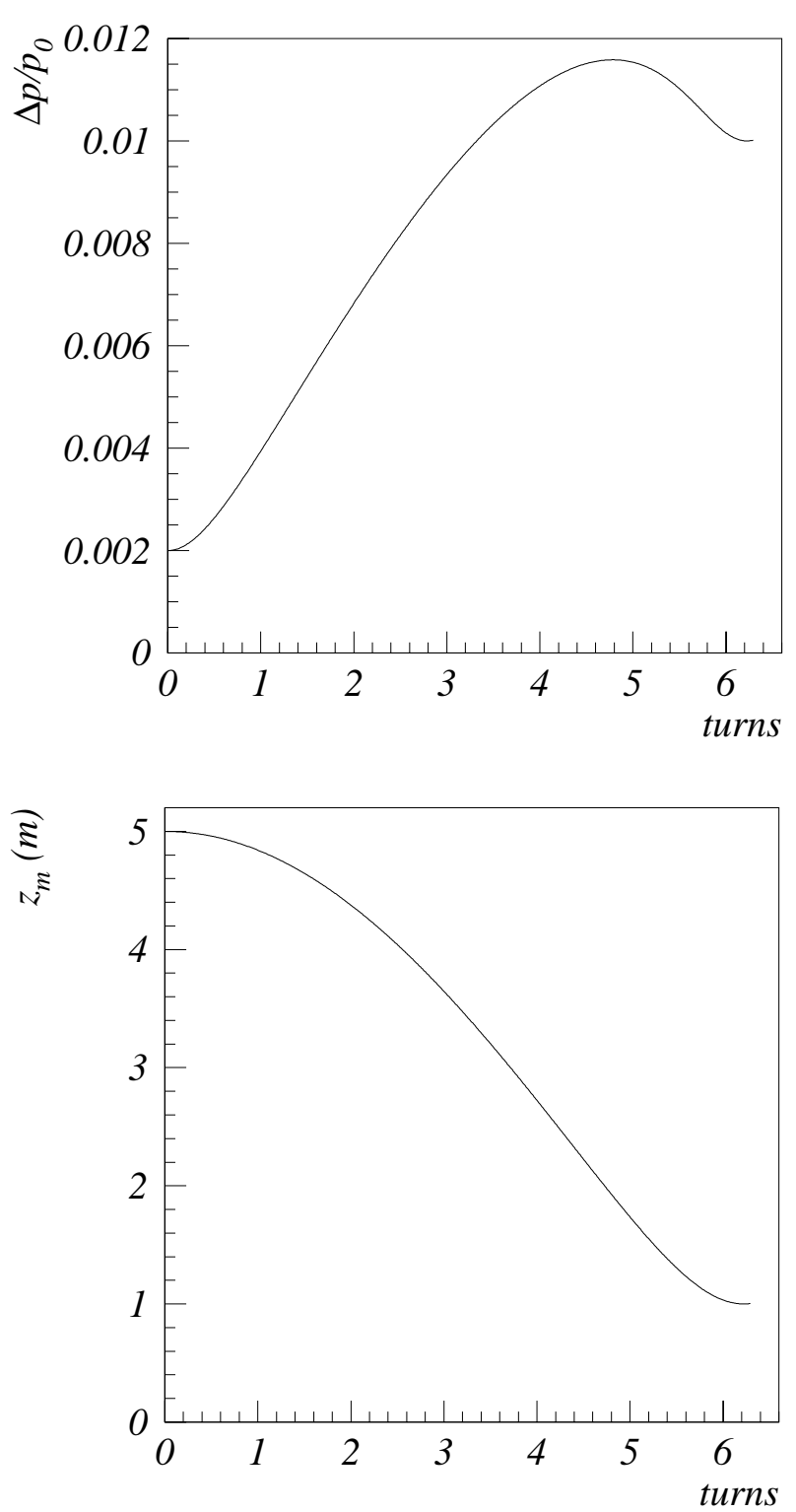

FIG. 3. Momentum spread and envelope for case (i), with $\eta=-0.1$.

(i) $\eta=-0.1$, with $\Sigma_{f}=1.14$, requiring $9.5 \mathrm{MV}$ and $\Delta_{\max } / \Delta_{f}=1.16$ (1.22) (see Fig. 3).

(ii) $\eta=-0.001$, with $\Sigma_{f}=114$, requiring $4.7 \mathrm{MV}$ and $\Delta_{\max } / \Delta_{f}=7.02$ (7.04) (see Fig. 4). It is seen that the reduced voltage is compensated by an increased number of turns and an unacceptably large coherent spread.

(iii) Using a two-step scheme starting with $\eta=-0.01$ and rotating to $\Delta_{\max } / \Delta_{f}=1.2$, we require $2.23 \mathrm{MV}$. Having reached the peak of the coherent momentum spread (turn 25) we complete the rotation (keeping the same voltage) at $\eta=-0.1$, which leads to the desired final length. In Fig. 5 we show the result, along with a $\pm 50 \%$ variation about the nominal $\eta$. Such a spread may result from the dependence of $\gamma_{t}$ on the incoherent tune shift or a combination of this and a dependence on the momentum
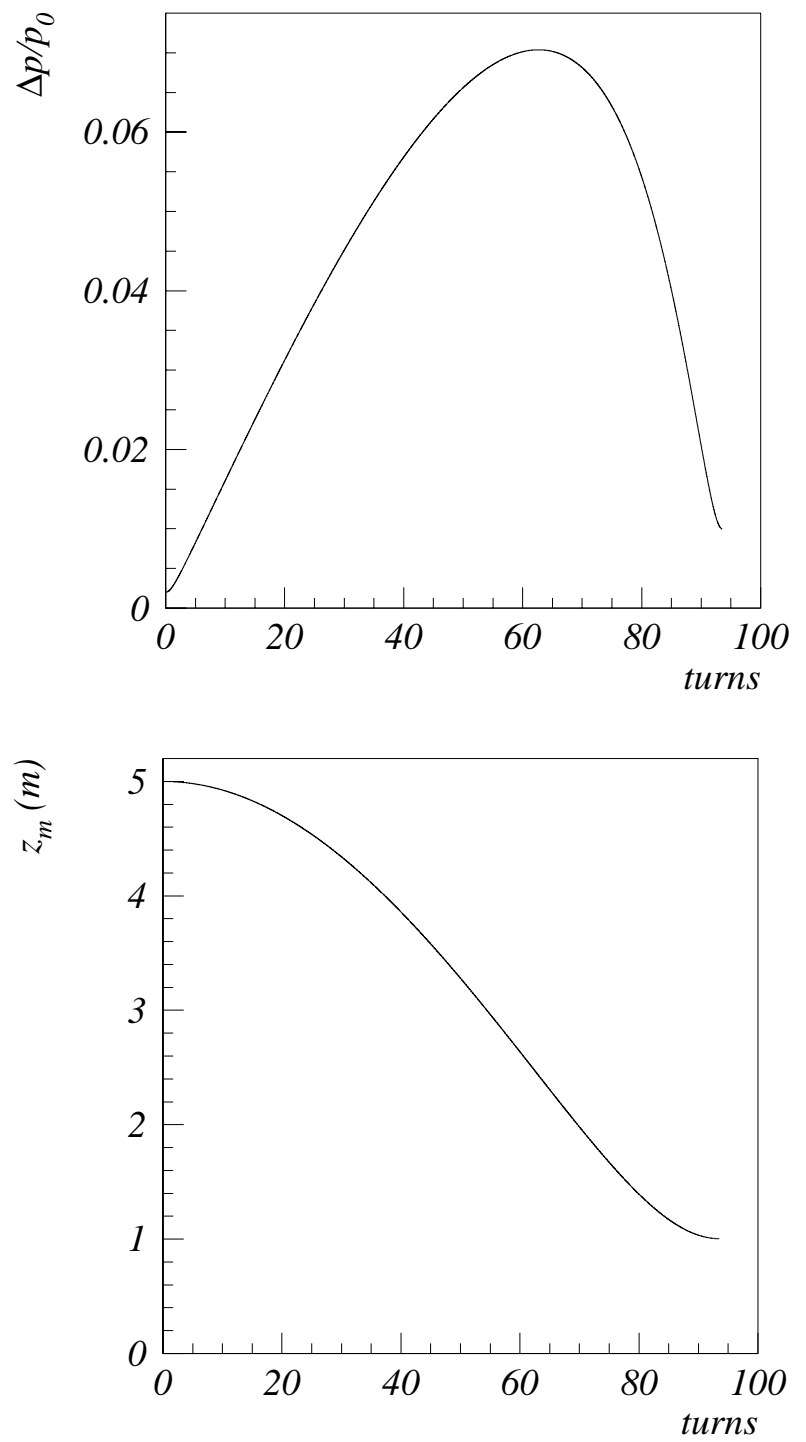

FIG. 4. Momentum spread and envelope for case (ii), with $\eta=-0.001$.

spread. The resulting final spread in bunch length and coherent momentum spread still appears tolerable. Significantly larger spreads in $\eta$ certainly need to be studied self-consistently with a simulation program. We also show in Fig. 5 the result for a complete compression at constant $\eta=-0.01$, which requires $5.1 \mathrm{MV}$ and leads to $\Delta_{\max } / \Delta_{f}=2.4$.

We notice that a two-step scheme jumping from $\eta=$ -0.001 to $\eta=-0.1$ would lower the required voltage to $0.95 \mathrm{MV}$, but the inevitable spread in $\gamma_{t}$ causes a significantly larger spread than was the case in example (iii). We also mention that the approximation in Eqs. (20) and (21) leads to 1.0 MV in this case; for the case of example (iii) the approximation significantly overestimates the correct value and predicts $3.1 \mathrm{MV}$, which is $39 \%$ too high. 

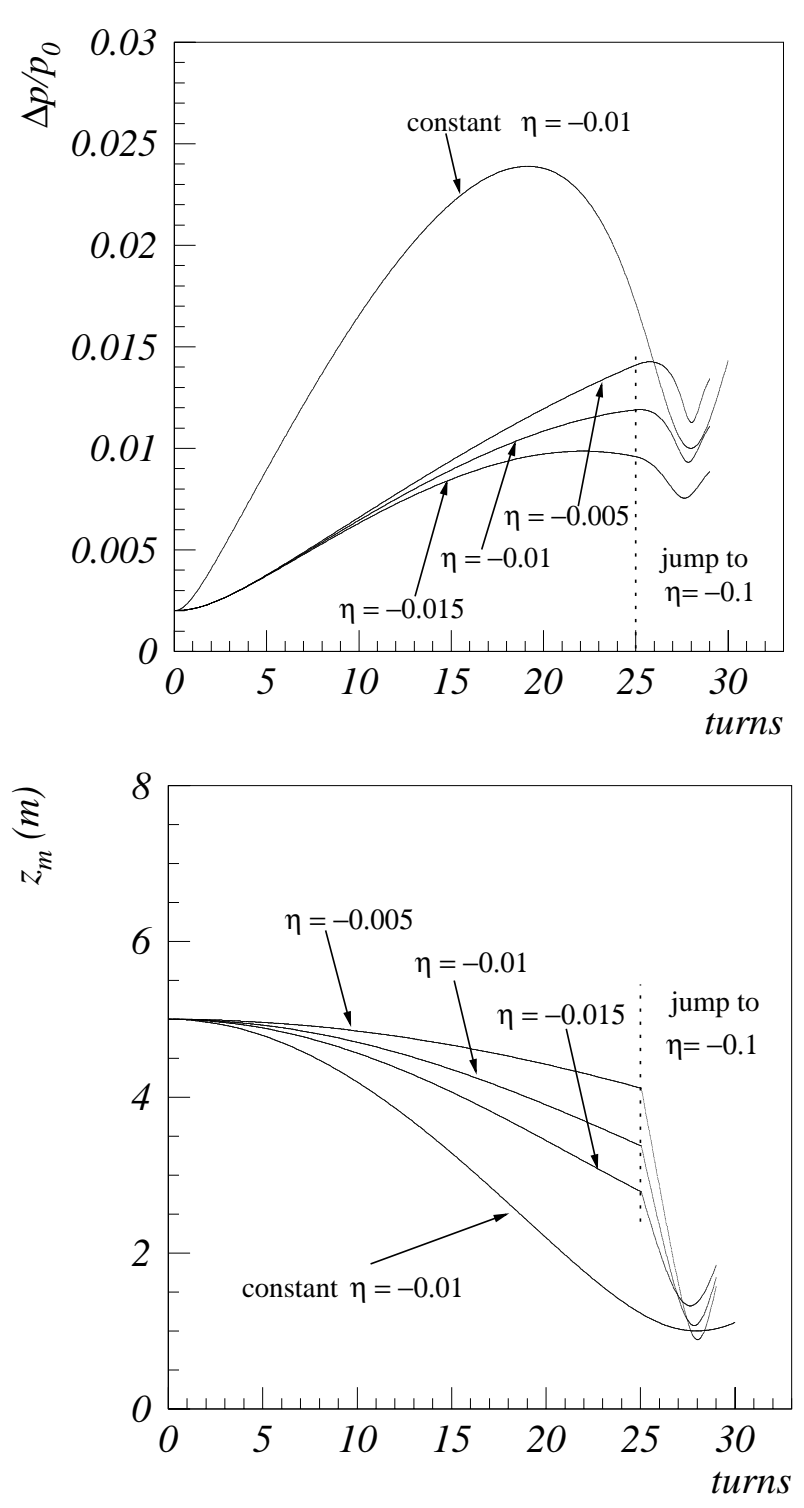

FIG. 5. Momentum spread and envelope for the $\eta$-jump case (iii), with $\eta=-0.01 /-0.1$.

\section{COMPUTER SIMULATION}

We have carried out a comparison with computer simulation using our code PATRIC. This is a particle-in-cell program solving the equations of motion in $3 \mathrm{D}$, whereas Poisson's equation is solved in cylindrical $(r-z)$ geometry assuming a perfectly conducting beam pipe [6]. PATRIC therefore treats the betatron motion in smooth approximation (symmetric in $x$ and $y$ ). Space charge is calculated correctly also for short bunches, where the $g$ factor approximation to the space charge electric field becomes incorrect. For long bunches we have found that the electric self-field on axis generated by a parabolic bunch in PATRIC agrees with the $g$-factor expression $g=$ $1-\left(r / R_{b}\right)^{2}+2 \ln \left(R_{p} / R_{b}\right)(r$ distance from axis) within $1 \%$ [6]. Note that the expression used in Eq. (2) is an average over a uniform transverse density. For the longitudinal motion in $z$ we have introduced the zero-order slip factor to model the real transverse lattice effect in lowest order according to $z^{\prime}=-\eta \delta p / p_{0}$.

Using parameters of the above example and $R_{p}=$ $0.1 \mathrm{~m}$, we have carried out a direct comparison with $10^{6}$ simulation particles on a $r-z$ grid of $32 \times 1024$ cells. We have reduced artificially the transverse betatron frequency to reduce the CPU time, but still allowed for enough betatron periods to keep a transverse averaging of the longitudinal force. The rotation is driven by a linear (in $z$ ) $\mathrm{rf}$ electric field calculated by using the envelope model. The case of moderate space charge $(\eta=-0.05)$ is compared with a strong space charge effect $(\eta=-0.005)$.

\section{A. Moderate space charge $\left(\eta=-0.05, \Sigma_{f}=2.28\right)$}

Figure 6 shows that there is quite good agreement between the envelope solution and an initial self-consistent parabolic bunch. The rms longitudinal emittance of the simulation grows by about $1 \%$, with the final momentum spread about 3\% larger and the bunch length about $2 \%$ shorter than for the envelope model. The slightly larger coherent spread indicates that the effective space charge in PATRIC is somewhat weaker than in the envelope model. This may be attributed to the fact that our simulation is in $r-z$, where finite beam pipe effects and the spread of the $g$ factor during betatron motion play a role. The final half-length of $1 \mathrm{~m}$ suggests, in fact, that the real space charge force is slightly reduced compared with the $g$-factor approximation (used to calculate the $\mathrm{rf}$ force), which explains qualitatively the slight additional compression.

We have compared this result with an initial rmsequivalent Gaussian bunch (same rms length and momentum width), starting with a Gaussian line density and Gaussian velocity distribution with constant width along the bunch, which shows a visible difference in the final part of compression. The final rms momentum spread is $4 \%$ and the rms bunch length $7 \%$ larger than for the initially parabolic bunch (see Fig. 7). Note that for parabolic bunches the rms bunch length is $z_{m} / \sqrt{5}$, and equally for the momentum width. We conclude that the above derived analytical expressions can be used with sufficient accuracy for nonparabolic bunches by using rms quantities multiplied by $\sqrt{5}$.

A more noticeable effect is seen in the scatter plots of Fig. 8, which show substantial momentum tails at final compression (note that we plot relative velocities given by $\beta c \eta \delta p / p$, with $\beta=0.923$ in our example). The turning point in the Gaussian line density implies that the space charge force is quite nonlinear and increases from the center to a maximum, beyond which it drops to zero. The phase space tails beyond this turning point are rotated faster than the core and move inside the bunch (with respect to $z$ ), where the profile flattens and space charge repulsion nearly vanishes. As a result, the velocity spread 

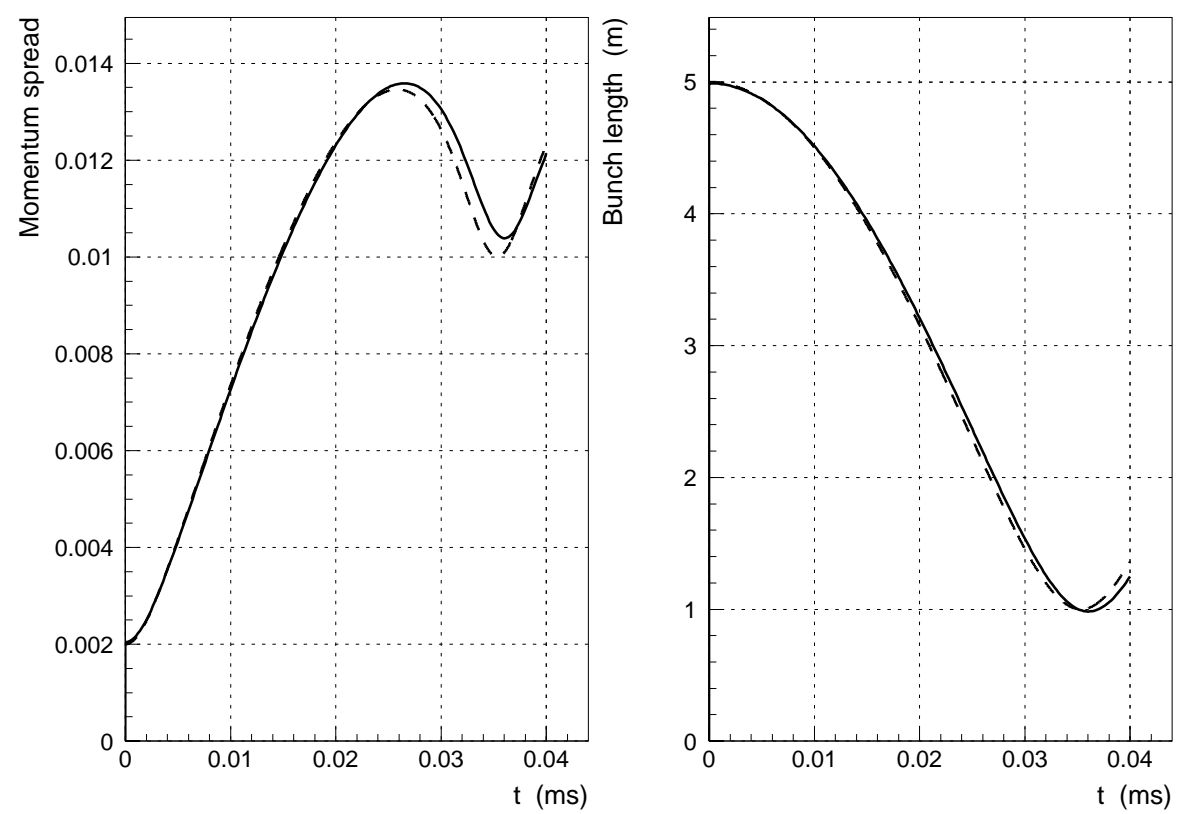

FIG. 6. Comparison of the envelope code result (dashed line) with a PATRIC particle-in-cell simulation for initial parabolic bunch with $\eta=-0.05$.
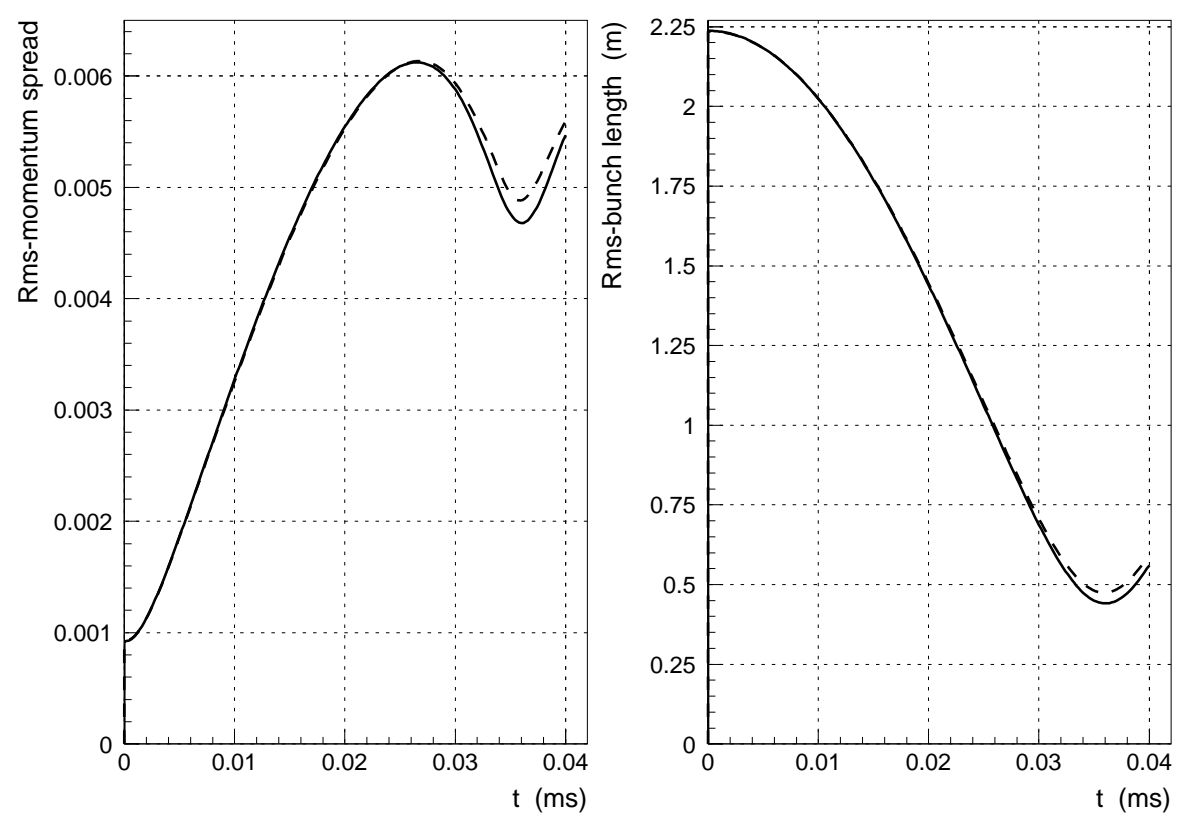

FIG. 7. Comparison of PATRIC simulation for initial parabolic and Gaussian bunch (dashed line) with $\eta=-0.05$.

gained in the early part of the rotation remains conserved for the tail population. We have estimated that approximately $5 \%$ of the total intensity is found in the tails beyond $\sqrt{5} z_{\text {rms }}$

\section{B. Strong space charge $\left(\eta=-0.005, \Sigma_{f}=22.8\right)$}

For the parabolic bunch the conservation of emittance is equally good (about $1 \%$ ), but the final momentum spread is found about $6 \%$ larger and the bunch length $6 \%$ shorter than in the envelope case. This trend is consistent with the significantly enhanced space charge effect. In Fig. 9 we compare the parabolic bunch with the initial Gaussian bunch and find an enhancement of the effect already observed for $\eta=-0.05$. The rms final momentum spread increases by $53 \%$, and the bunch length increases by $13 \%$. The momentum tails are significantly more extended according to the larger coherent $\Delta$; yet they contain again approximately $5 \%$ of the total intensity beyond $\sqrt{5} z_{\mathrm{rms}}$ (see Fig. 10). 

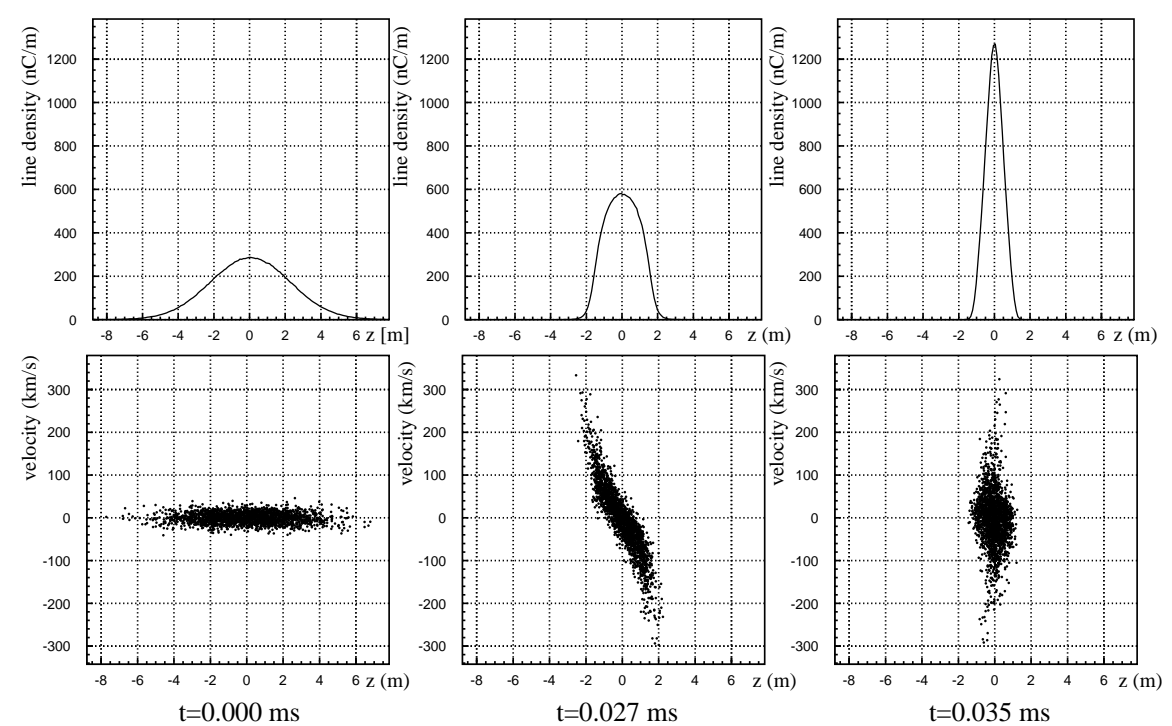

FIG. 8. Line density and scatter plot of longitudinal phase space for Gaussian bunch with $\eta=-0.05$ at different phases of compression.
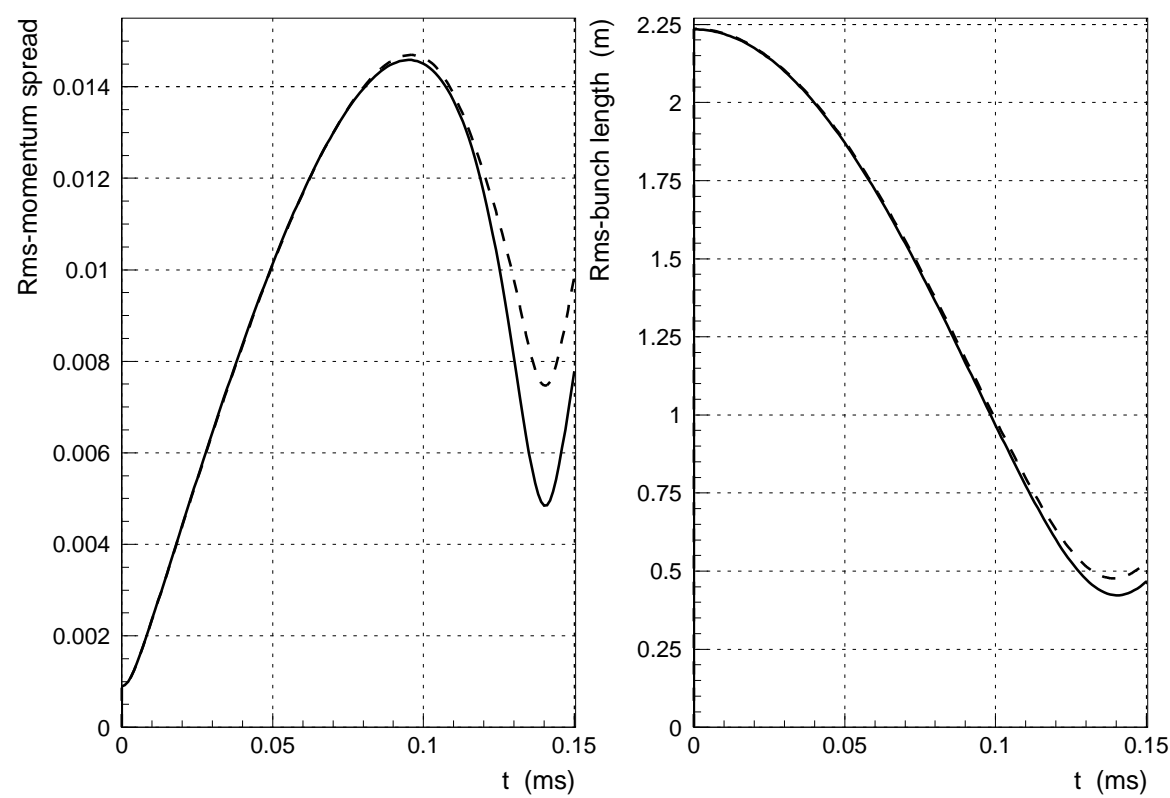

FIG. 9. Comparison of PATRIC simulation for initial parabolic and Gaussian bunch with $\eta=-0.005$.

We have compared this result with a semiparabolic bunch using an initial parabolic line density, but Gaussian velocity distribution of constant width along the bunch, and find excellent agreement with the parabolic bunch (better than $0.3 \%$ everywhere). Here it should be noted that the line density remains parabolic during most of the compression history and changes only close to completion of the $90^{\circ}$ rotation when the Gaussian velocity distribution folds visibly into real space. The final longitudinal distribution is box shaped as is shown in the scatter plots of Fig. 11, with practically no momentum tails.

\section{Estimates for momentum tail}

The maximum extent $\Delta_{\text {tail }}$ of the momentum tails can be estimated analytically by assuming that the bunch ends see practically no space charge force during the first part of the rotation. They are then rotated by the full $\mathrm{rf}$ force given from Eq. (15) until they reach a maximum momentum deviation

$$
\Delta_{\text {tail }} \approx b_{l} \Delta_{f}\left(1+\frac{\Sigma_{f}}{(1+\chi)}\right)^{1 / 2}
$$




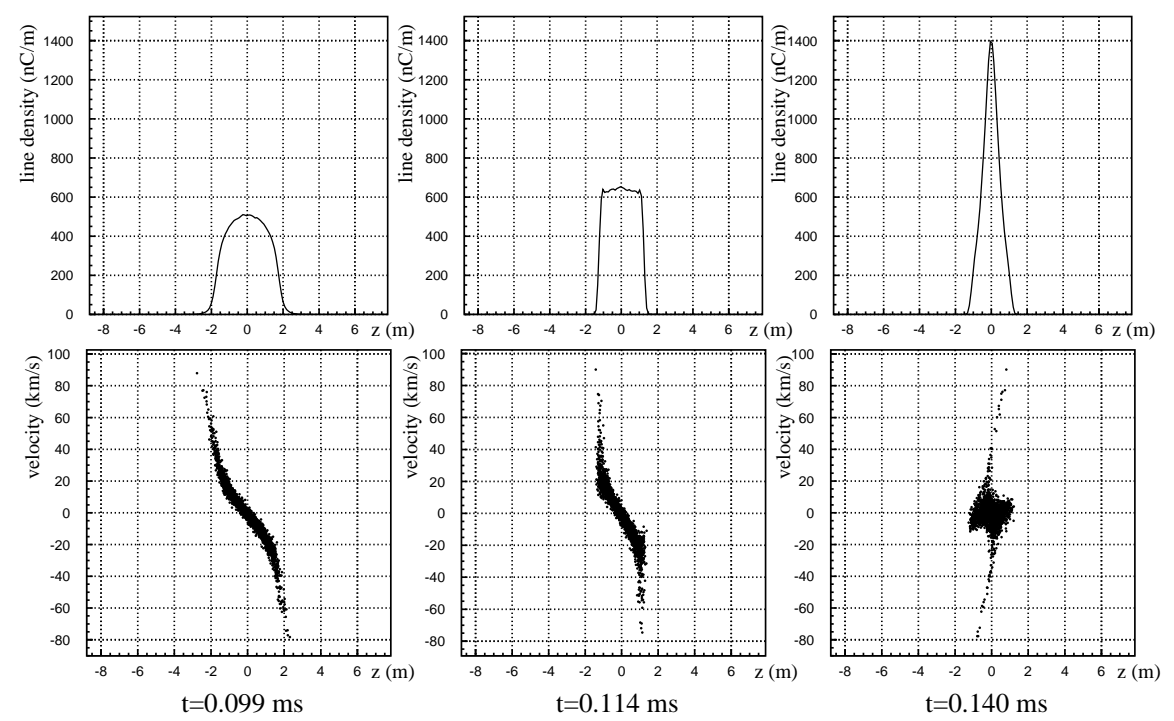

FIG. 10. Line density and scatter plot of longitudinal phase space for Gaussian bunch with $\eta=-0.005$.
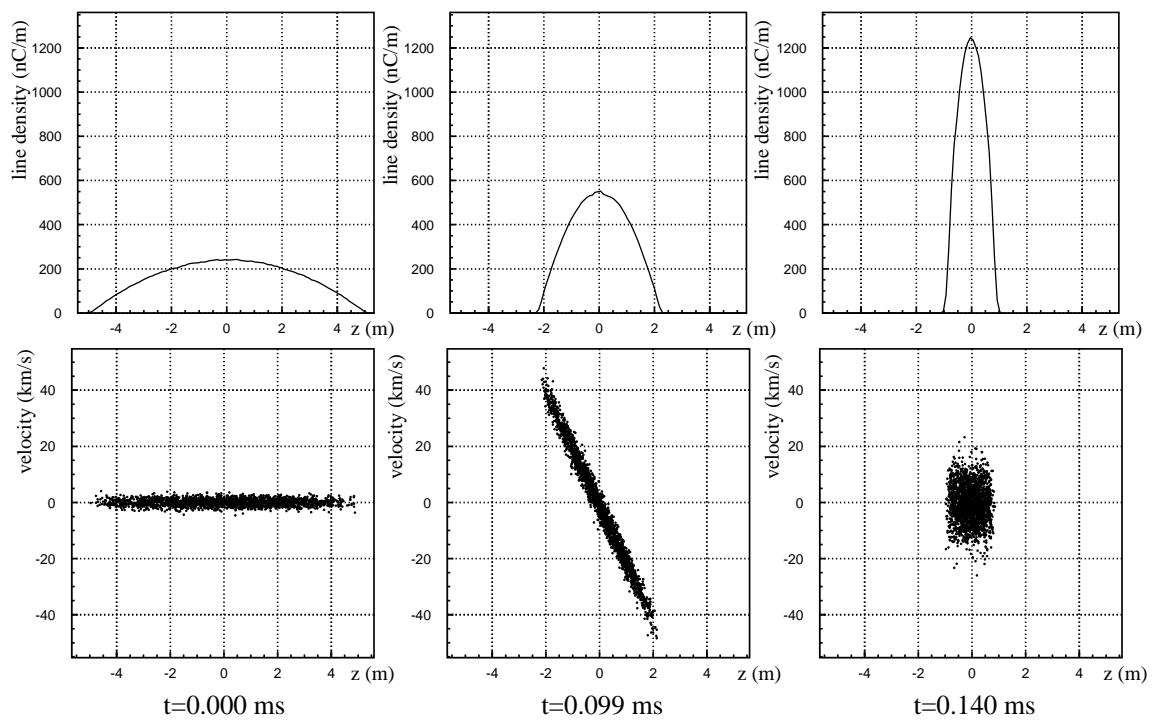

FIG. 11. Line density and scatter plot of longitudinal phase space for a semiparabolic bunch with $\eta=-0.005$.

where $\Delta_{f}$ is the ideal final momentum spread of the rms equivalent parabolic bunch (i.e., $\sqrt{5} \Delta_{\mathrm{rms}}$ ). The factor $b_{l}$ should take into account the effectively longer bunch length of the rms equivalent nonparabolic bunch, which leads to a correspondingly increased momentum gain ( $b_{l} \approx 1.4$ for a Gaussian bunch). Applying this expression to the above examples we calculate a maximum extent of $330 \mathrm{~km} / \mathrm{s}$ for the case of Fig. 8 and $87 \mathrm{~km} / \mathrm{s}$ for the case of Fig. 10, which is well confirmed by the scatter plots. We expect that Eq. (23) applies generally to nonparabolic bunches with a turning point in the line density.

\section{CONCLUSION}

By using energy conservation we have derived analytical expressions for the space charge effect during compression. From a comparison with computer simulation we conclude that these expressions (as well as the envelope equations) can be applied also to rms-equivalent nonparabolic bunches by using $z_{m}=\sqrt{5} z_{\text {rms }}$ (and similar for the momentum spread), provided that space charge is not too strong. We also confirm by simulation that short bunch length effects are small for the considered parameters. 
The attractiveness of using small $\eta$ is, however, limited in practice if space charge dominates the rf voltage and leads to a significant coherent momentum spread prior to completion of the compression. In our simulation of Gaussian bunches we have found significant momentum tails in the compressed bunches, containing typically $5 \%$ of the total intensity, even for moderately large space charge. From a practical point of view they might be beyond the momentum acceptance of the ring (or extraction beam line) and therefore add to the beam loss and activation problem in a high power ring. The practical feasibility of the proposed two-step scheme, by jumping from small to larger $|\eta|$ before the end of compression (thus avoiding the most serious space charge issues), needs further examination. Further complications arising from a spread in $\eta$ due to a betatron tune shift and spread caused by space charge, as well as higher order terms in $\Delta$, will have to be considered in future studies.

[1] D. Neuffer, IEEE Trans. Nucl. Sci. 26, 3031 (1979).

[2] L. Smith, in Proceedings of the Summer Study of Heavy Ions for Inertial Fusion, Berkeley, CA, 1976 (LBL Report No. LBL-5543, 1976), p. 77.

[3] I. Hofmann, in Proceedings of the Symposium on Accelerator Aspects of Heavy Ion Fusion, Darmstadt, 1982 (Gesellschaft für Schwerionenforschung, Darmstadt, 1982), p. 181.

[4] M. Reiser, Theory and Design of Charged Particle Beams (Wiley, New York, 1994), p. 432.

[5] A.G. Ruggiero and V.G. Vaccaro, CERN Report No. CERN-ISR-TH/68-33, 1968 (unpublished).

[6] I. Hofmann and G. Kalisch, Phys. Rev. E 53, 2807 (1996). 